Check for updates

Cite this: Phys. Chem. Chem. Phys., 2017, 19, 16283

DOI: $10.1039 / c 7 c p 90124 k$

\section{Correction: A review of carrier thermoelectric- transport theory in organic semiconductors}

\author{
Nianduan Lu, ${ }^{\text {ab }}$ Ling Li*ab and Ming Liu ${ }^{\text {ab }}$ \\ Correction for 'A review of carrier thermoelectric-transport theory in organic semiconductors' by \\ Nianduan Lu et al., Phys. Chem. Chem. Phys., 2016, 18, 19503-19525.
}

rsc.li/pccp

In the published paper, we described two definitions of the conductivity. One is defined by Mott (see eqn (7), $\left.\sigma=-\int \sigma(E) \frac{\partial f}{\partial E} \mathrm{~d} E\right)$. The other is defined as the Kubo-Greenwood type conductivity (see eqn (22), $\sigma=\int \sigma(E) \mathrm{d} E$ ). The definition of conductivity for both eqn (7) and (22) is equivalent. In order to better distinguish these two definitions, we change eqn (7) to $\sigma=-\int \sigma_{\mathrm{E}} \frac{\partial f}{\partial E} \mathrm{~d} E$, where $\sigma_{\mathrm{E}}$ is the Mott-type conductivity. At the same time, we change eqn (8) to $\sigma=e \int g(E) \mu(E) f(E) \mathrm{d} E$, and eqn (9) to $\sigma(E)=e g(E) \mu(E) f(E)$, in order to be consistent with eqn (22).

Additionally, we remove the differential term $\mathrm{d} E$ in eqn (23), so that eqn (23) should be modified to $\sigma(E)=e g(E) \mu(E) f(E)$.

Furthermore, in the published paper, $R^{3}$ was omitted in eqn (44). Therefore, the parameter $R^{3}$ should be added in eqn (44), as follows:

$$
P\left(Z_{\mathrm{m}} \mid E_{\mathrm{i}}\right)=\frac{4 \pi R^{3}}{3(2 \alpha)^{3}} \times\left\{\begin{array}{l}
\int_{\varepsilon_{\mathrm{f}}}^{\varepsilon_{\mathrm{i}}}\left(S_{\mathrm{c}}-\varepsilon_{\mathrm{j}}+\varepsilon_{\mathrm{f}}\right)^{3} g\left(\varepsilon_{\mathrm{j}}\right) \mathrm{d} \varepsilon_{\mathrm{j}}+\int_{\varepsilon_{\mathrm{i}}}^{S_{\mathrm{c}}+\varepsilon_{\mathrm{f}}}\left(S_{\mathrm{c}}-\varepsilon_{\mathrm{j}}+\varepsilon_{\mathrm{f}}\right)^{3} g\left(\varepsilon_{\mathrm{j}}\right) \mathrm{d} \varepsilon_{\mathrm{j}}+\int_{\varepsilon_{\mathrm{i}}-S_{\mathrm{c}}}^{\varepsilon_{\mathrm{f}}}\left(S_{\mathrm{c}}-\varepsilon_{\mathrm{i}}+\varepsilon_{\mathrm{j}}\right)^{3} g\left(\varepsilon_{\mathrm{j}}\right) \mathrm{d} \varepsilon_{\mathrm{j}}, \varepsilon_{\mathrm{i}}>\varepsilon_{\mathrm{f}} \\
\int_{\varepsilon_{\mathrm{i}}}^{\varepsilon_{\mathrm{f}}}\left(S_{\mathrm{c}}+\varepsilon_{\mathrm{i}}-\varepsilon_{\mathrm{f}}\right)^{3} g\left(\varepsilon_{\mathrm{j}}\right) \mathrm{d} \varepsilon_{\mathrm{j}}+\int_{\varepsilon_{\mathrm{f}}-S_{\mathrm{c}}}^{\varepsilon_{\mathrm{i}}}\left(S_{\mathrm{c}}+\varepsilon_{\mathrm{i}}-\varepsilon_{\mathrm{f}}\right)^{3} g\left(\varepsilon_{\mathrm{j}}\right) \mathrm{d} \varepsilon_{\mathrm{j}}+\int_{\varepsilon_{\mathrm{f}}}^{\varepsilon_{\mathrm{i}}+S_{\mathrm{c}}}\left(S_{\mathrm{c}}-\varepsilon_{\mathrm{j}}+\varepsilon_{\mathrm{i}}\right)^{3} g\left(\varepsilon_{\mathrm{j}}\right) \mathrm{d} \varepsilon_{\mathrm{j}}, \varepsilon_{\mathrm{i}}<\varepsilon_{\mathrm{f}}
\end{array}\right.
$$

We thank Dario Narducci for finding this error.

The Royal Society of Chemistry apologises for these errors and any consequent inconvenience to authors and readers.

\footnotetext{
${ }^{a}$ Key Laboratory of Microelectronic Devices \& Integrated Technology, Institute of MicroElectronics of the Chinese Academy of Sciences, No. 3, Bei-Tu-Cheng West Road, Beijing 100029, China. E-mail: lingli@ime.ac.cn

${ }^{b}$ Jiangsu National Synergetic Innovation Center for Advanced Materials (SICAM), Nanjing 210009, China
} 